\title{
Grand challenges in condensed matter physics: from knowledge to innovation
}

\author{
Evgeny Y. Tsymbal* and Peter A. Dowben \\ Department of Physics and Astronomy, Nebraska Center for Materials and Nanoscience, University of Nebraska, Lincoln, NE, USA \\ *Correspondence: tsymbal@unl.edu
}

Edited by:

Alex Hansen, Norwegian University of Science and Technology, Norway

Keywords: condensed matter physics, grand challenges, strongly correlated systems, topological insulators, skyrmions, nanotechnology, spintronics

Condensed Matter Physics (CMP) explores the fundamental properties of matter and their origins resulting from the interactions of a large number of atoms and electrons. The intricate nature of these interactions results in properties and associated phenomena that often hint at a rich vein of underlying physics. Although the perspective is changing constantly with new discoveries, the basic challenges in CMP are to predict and observe new phenomena and elucidate novel properties of materials often pushing at the frontiers of quantum mechanics [1].

CMP is also a field which stimulates technological innovation that revolutionizes modern society. For more than five decades, the engine of CMP has largely been driven by semiconductor industry. Probably the most notable example is the invention of the transistor which was recognized by the 1956 Nobel Prize in Physics given to William Shockley, John Bardeen, and Walter Brattain. The transistor-a basic building block of modern electronic devices-was a result of innovative research in the field of semiconductors. The transistor and the invention of the integrated circuit in 1958 was the starting point for exponential increase in the computational power known as Moore's law [2]. There is a persistent interplay between the fundamental science and technological applications which provides breadth to CMP [3].

One cannot possibly give full justice to the entire range of CMP problems that now command the attention of the condensed matter and materials physics community. Therefore, rather than even try, in this short essay we point out a few fundamental problems of major importance whose solution would further expand our understanding and Knowledge, while also mentioning some emerging functional properties of materials where the associated potential applications could foster the technological Innovation.

\section{FROM KNOWLEDGE}

In condensed matter, striking phenomena emerge from interactions between the constituent particles and the interplay between coupled degrees of freedom. The quantum-mechanical nature of these interactions makes condensed matter phenomena non-trivial and often counterintuitive. Superconductivity is one of the extraordinary examples of such a behavior.

Superconductivity is the property of a material to carry an electrical current with no dissipation of energy. First discovered by Onnes in 1911, superconductivity had no explanation for nearly half a century. Only in 1957 did Bardeen, Cooper, and Schrieffer (BCS) elucidate superconductivity as an effect caused by condensation of Cooper pairs into a boson-type state [4]. The BCS theory provided a consistent understanding of this phenomenon in metals where the superconducting transition occurs at cryogenic temperatures. However, 20 years later Karl Müller and Johannes Bednorz discovered cuprate superconductors which had much higher transition temperatures [5]. Properties of these high-temperature superconductors did not match the BCS theory based on electron pairing due to electron-phonon interaction. So far, no generally accepted theory of high-temperature superconductivity exists, while the recent discovery of superconductivity in iron pnictides may also indicate a non-conventional mechanism. Microscopic understanding of superconductivity in these compounds remains a challenge for CMP.
High-temperature superconductivity leads directly to one of the immense problems in CMP-understanding the properties of strongly correlated electronic systems. The key feature of the strongly correlated systems is that their electronic behavior cannot be described adequately in terms of the non-interacting particles picture. For example, due to poor screening, the interaction energy between valence electrons in doped complex oxide materials often overcomes their kinetic energy, resulting in a strongly coupled many-body ground state. Similarly, actinides and lanthanides are characterized by the localized $f$ levels, which are often hybridized with $s$, $p$, and $d$ states, leading to the strong on-site and inter-site Coulomb interactions where a single-particle wave function is a poor approximation. Properties of the strongly correlated systems are controlled by the competition between different electronic phases, often characterized by various types of charge and spin ordering and involving different length and energy scales. This competition leads to intrinsic inhomogeneities (e.g., phase separation) in these materials and intricate phase diagrams. As a result of the strong electron-electron correlations, these materials are extremely sensitive to external perturbations and display a variety of interesting properties, such as hightemperature superconductivity, colossal magnetoresistance, metal-insulator transitions, etc. Condensed matter systems are intrinsically many body, and while the quantum mechanical single particle picture (plus a whole host of perturbations and corrections) works surprisingly well in explaining much of the phenomena observed, we have to admit that the solutions constructed are often ad hoc, and 
only vaguely address the many body electronic structure. Reformulating solid-state theory to adequately describe strongly correlated systems is another grand challenge in CMP.

The emergence of non-trivial cooperative phenomena in CMP is often driven by the interplay between well-known constituents; yet the collective behavior may be strikingly dissimilar and often unexpected. This is the case for the new quantum states of matter recently discovered. For example, the fractional quantum Hall state represents a peculiar electronic liquid, where an added electron breaks up into new particles, each carrying an exact fraction of the electron charge $[6,7]$. Perpendicular-to-the-plane magnetic field applied to a two-dimensional electron system breaks up the otherwise continuous distribution of electron energies into discrete states known as Landau levels. At a sufficiently high magnetic field, all electrons lie on the lowest Landau level precisely at the same kinetic energy. Coulomb interaction between electrons dominates their behavior, representing a strongly interacting quantum system: a new strongly-correlated state of matter.

Critical phenomena and phase transitions are an important constituent of modern CMP. Phase transition refers to the transformation of thermodynamic system from one phase or state of matter to another. Systems undergoing a phase transition exhibit critical behavior, where several properties such as specific heat and susceptibility diverge. Some transitions, such as the paramagnetic-ferromagnetic phase transition, occur "continuously" so that close to the critical temperature it becomes impossible to assign the state to either of the two phases involved, and the system exhibits a new critical behavior. Continuous phase transitions are described by the Ginzburg-Landau theory [8], which exploits the so-called mean field approximation. However, a number of phase transitions, such as metal-insulator transitions, do not follow the GinzburgLandau approach. Phase transitions, especially in strongly correlated systems, is an active area of research.

The complexity of physical phenomena is also evident in soft matter-a subfield of condensed matter which involves physical states that are easily deformed by thermal or mechanical means. The soft matter involves a variety of organic materials such as polymers, colloids, and liquid crystals. An important common feature of these materials is that the most interesting properties of soft matter emerge directly from its atomic or molecular constituents. The complexity and diversity of physical behaviors of soft matter is due to the fact that the macroscopic properties of these materials is determined by interactions at the mesoscopic scale which, on one hand, involves a large number of atoms and molecules, but, on the other hand, is much smaller than the macroscopic scale. Although soft materials emerge in different forms, many of their physical and chemical properties have common origins, such as multiple degrees of freedom, weak interactions between structural constituents, and a subtle balance between contributions to the free energy from entropy and enthalpy. All these materials are sensitive to external conditions and characterized by sizable thermal fluctuations, emergence of metastable states, and a wide variety of forms.

Several significant efforts in CMP are related to the exploration of materials properties at the nanometer scale. Materials properties at distances of 100 nanometers or less deviate significantly from the bulk properties leading to new phenomena and functional characteristics. As dimensions shrink to the nanoscale, surface and interface properties dominate the material behavior: according to Kroemer (a 2000 Nobel Prize Laureate) "The interface is the device." The old view: "God made the bulk; the surface was invented by the devil," articulated by Wolfgang Pauli (the 1945 physics Nobel Prize Laureate), must be somehow overcome in the culture of CMP.

An important class of nanoscale objects is based on an intrinsically twodimensional material_-graphene-a sheet of carbon atoms bonded in a honeycomb pattern [9]. Groundbreaking experiments on graphene were acknowledged by the 2010 Nobel Prize in Physics given to Geim and Novoselov. The growth of single crystalline graphene with the controllable number of layers still remains a challenge, although a significant progress has been achieved in the recent years. The intensity of research on graphene is now enormous, and has been expanded now to other structurally similar two-dimensional materials, such as hexagonal boron nitride, $\mathrm{MoS}_{2}$, and $\mathrm{WSe}_{2}$, opening a vista of reduced dimensional systems with a range of spinorbit coupling effects from barely none at all to quite significant.

There are a number of systems where key properties (conductivity, spin-orbit coupling, spin current) are protected by topology. Among them are the topological insulators-electronic materials that have a bulk band gap like ordinary insulators, but exhibit conducting states on their edge or surface [10]. Unlike band insulators that can also support conductive surface states $[11,12]$, the surface states of topological insulators are special due to being symmetry protected by time reversal symmetry. The two-dimensional topological insulator can be considered as a quantum spin Hall state, where helical edge states interconnect spin and momentum of the carriers and lead to quantized conductance of charge and spin [13]. Indeed, the quantum spin Hall effect in $\mathrm{HgTe} /(\mathrm{Hg}, \mathrm{Cd}) \mathrm{Te}$ quantum wells may point to fundamentally new quantum state of matter [14]. Other topological objects in condensed matter (though extensively discussed outside condensed matter for decades) are skyrmions [15] —a twisted vertex configuration of a two-dimensional ferromagnet. Skyrmions may form spontaneously in magnetic materials though a Dzyaloshinskii-Moriya interaction without the assistance of external fields or the proliferation of defects [16]. Magnetic skyrmions are typically as small as a $1 \mathrm{~nm}$ in size and arrange in two-dimensional lattices [17]. They can be created and erased in a controlled way using local spin-polarized currents from a scanning tunneling microscope [18], which opens the potential for their use in informationstorage concepts.

\section{TO INNOVATION}

Discoveries of novel phenomena and materials properties driven by fundamental studies in CMP often are realized in industrial innovations. We have already mentioned the discovery of the transistor which has revolutionized the semiconductor industry. Another prominent example (though not of the same scope as semiconductor technology) is spintronics where 
both electron charge and spin contribute to the functional properties of electronic and data storage devices [19].

The spin nature of electron transport lies in the heart of modern magnetic data storage technologies, such as computer hard drives and magnetic random access memories (MRAMs), and illustrates the progress from Mott's efforts in 1936 to understand the importance of the electron spin in electrical conductivities of the transition metals and their alloys $[20,30]$. Current spintronics technology employs a magnetoresistive device, a metallic spinvalve or a magnetic tunnel junction, that consists of two ferromagnetic electrodes separated by a thin spacer layer of nonmagnetic material. The flow of carriers through the device is determined by the direction of their spin relative to the magnetization of the device's electrodes resulting in giant magnetoresistance (GMR) $[21,22]$ if the spacer is metallic and in tunneling magnetoresistance (TMR) [23, 24] if the spacer is insulating. The discovery of GMR was recognized by the 2007 Nobel Prize in Physics given to Fert and Grünberg, for the impact of GMR on the technology that is used to read data on hard disks.

Spintronics has evolved into a vigorous field of research involving, e.g., the search for magnetic dilute semiconductors with the Curie temperature above the room temperature, spin injection in metals and semiconductors, spin-dependent thermal effects, and current driven spintransfer torques. The latter is especially relevant to modern technologies, since the spin transfer torques can be used to switch the magnetization direction, thus providing an advanced tool to write the information in MRAMs. The spin Hall effect, which was predicted more than 40 years ago [25] but experimentally demonstrated only recently [26,27], may also become an ingredient in device functionalities. Driven by the spin-orbit interaction, an electrical current induces a transverse spin current and results in the appearance of spin accumulation on the lateral surfaces of a current-carrying sample. The spin current driven by the spin Hall effect opens up interesting possibilities for spin-torque switching of magnetization of a ferromagnet at room temperature [28].
The emergent phenomena in CMP will likely be decisive in extending or altering the shape of the International Technology Roadmap for Semiconductors, which could help in maintaining the Moore's law [2]. Reducing CMOS transistor dimensions to cater for higher speed and higher packing density leads to the increasing source-drain leakage and hence to the escalating power dissipation. Overcoming this problem requires novel materials and approaches for device operation. Various schemes have been proposed involving voltage control of magnetization, graphene field effect transistors, non-local spin valves, ferroelectric tunnel junctions, and others. Special emphasis is made on non-volatile operation of these devices which puts forward again an emphasis on ferromagnetism and ferroelectricity, long standing important subfields of CMP. The challenges are vast: the stated goals are non-volatile memory and logic with switching energy less that $10^{-2}$ fJ, switching speeds of $1 \mathrm{GHz}$ or better and stability over $10^{16}$ switches.

CMP plays an important role in meeting energy demands of the modern society. Affordable energy, mainly from fossil fuels, has enabled technological and human development. However, the deepening energy crisis requires a coordinated effort to harness alternative resources of renewable energy to create more energy-efficient and environmentally friendly technologies. The exploration of new materials is a necessary condition to effectively address the present and future grand challenges in energy generation, conversion, storage, and efficient utilization. The role of CMP in these challenges is hard to overestimate [3].

One example is the effort in developing cheaper and more efficient solar cellselectrical devices converting the energy of light into electricity by the photovoltaic effect. Another example is solidstate lighting, which has made remarkable progress in recent years. There are other energy-related technologies where CMP has contributed and continues contributing significantly. Among them are the light-assisted water decomposition which directly converts solar power to hydrogen by means of photocatalysis and the development of thermoelectric materials which can be used for conversion between thermal and electrical energy.

Obviously the above examples are not exhaustive but provide a flavor of the breadth of the CMP research. The future looks bright for the development of new materials to obtain more and greater functionality and enhanced performance. Concerning the fundamental challenges, such as an adequate description of strongly correlated electronic systems, there is the glimmer of new knowledge that now beckon us toward a much better understanding of not only CMP itself, but also other disciplines, such as quantum mechanics generally. Moreover, there are analogies between phenomena occurring in condensed matter, such as formation of topological defects or symmetry-breaking phase transitions, and processes intrinsic to early universe cosmology [29]. This relevance of CMP to other disciplines emphasizes the stature of the field and the significance of its grand challenges.

\section{ACKNOWLEDGMENTS}

The authors acknowledge the NSF funded Nebraska Materials Research Science and Engineering Center (Grant No. DMR0820521) for supporting fruitful research and collaboration of the authors during the last 11 years and the NRI funded Center for NanoFerroic Devices for stimulating engagement of the authors in the technologically challenging research. The authors are grateful to Verona Skomski for her assistance in preparation of the manuscript.

\section{REFERENCES}

1. Kohn W. An essay on CMP in the twentieth century. Rev Mod Phys. (1999) 71:S59-S77. doi: 10.1103/RevModPhys.71.S59

2. Moore GE. Cramming more components onto integrated circuits. Proc IEEE (1998) 86:82-5. doi: 10.1109/JPROC.1998.658762

3. Committee on CMMP 2010, Solid State Sciences Committee, Board on Physics and Astronomy, Division on Engineering and Physical Sciences, National Research Council. Condensed-Matter and Materials Physics: The Science of the World Around Us. Washington, DC: National Academies Press (2007). 259.

4. Bardeen J, Cooper LN, and Schrieffer JR. Theory of superconductivity. Phys Rev. (1957) 108:1175-204. doi: 10.1103/PhysRev.108. 1175

5. Bednorz JG, and Mueller KA. Possible high $\mathrm{T}_{C}$ superconductivity in the $\mathrm{Ba}-\mathrm{La}-\mathrm{Cu}-\mathrm{O}$ 
System. Z Phys B (1986) 64:189-93. doi: 10.1007/BF01303701

6. Tsui DC, Stormer HL, and Gossard AC. Two-dimensional magnetotransport in the extreme quantum limit. Phys Rev Lett. (1982) 48:1559-62. doi: 10.1103/PhysRevLett. 48.1559

7. Laughlin RB. Anomalous quantum hall effect: an incompressible quantum fluid with fractionally charged excitations. Phys Rev Lett. (1983) 50:1395-8. doi: 10.1103/PhysRevLett. 50.1395

8. Ginzburg VL, and Landau LD. On the theory of superconductivity. Zh Eksp Teor Fiz. (1950) 20:1064-82.

9. Novoselov KS, Geim AK, Morozov SV, Jiang D, Zhang Y, Dubonos SV, et al. Electric field effect in atomically thin carbon films. Science (2004) 306:666-9. doi: 10.1126/science. 1102896

10. Kane CL, and Mele EJ. Z2 topological order and the quantum spin hall effect. Phys Rev Lett. (2005) 95:146802. doi: 10.1103/PhysRevLett.95.146802

11. Tamm I. On the possible bound states of electrons on a crystal surface. Phys Z Sov Un. (1932) 1:733.

12. Shockley W. On the surface states associated with a periodic potential. Phys Rev. (1939) 56:317-23. doi: 10.1103/PhysRev.56.317

13. Bernevig BA, and Zhang SC. Quantum spin hall effect. Phys Rev Lett. (2006) 96:106802. doi: 10.1103/PhysRevLett.96.106802

14. König $M$, Wiedmann $S$, Brüne $C$, Roth $A$, Buhmann H, Molenkamp LW, et al. Quantum spin hall insulator state in HgTe quantum wells. Science (2007) 318:766-70. doi: 10.1126/science. 1148047

15. Skyrme T. A unified field theory of mesons and baryons. Nucl Phys. (1962) 31:556-69. doi: 10.1016/0029-5582(62)90775-7
16. Rößler UK, Bogdanov AN, and Pfleiderer C. Spontaneous skyrmion ground states in magnetic metals. Nature (2006) 442:797-801. doi: 10.1038/nature05056

17. Mühlbauer S, Binz B, Jonietz F, Pfleiderer C, Rosch A, Neubauer A, et al. Skyrmion lattice in a chiral magnet. Science (2009) 323:915-9. doi: $10.1126 /$ science. 1166767

18. Romming N, Hanneken C, Menzel M, Bickel JE, Wolter B, von Bergmann K, et al. Writing and deleting single magnetic skyrmions. Science (2013) 341:636-9. doi: 10.1126/science.1240573

19. Tsymbal EY, and Žutić I. (eds.). Handbook of Spin Transport and Magnetism. Boca Raton, FL: CRC press (2011). 808.

20. Mott NF. The resistance and thermoelectric properties of the transition metals. Proc R Soc. (1936) 156:368-82.

21. Baibich MN, Broto JM, Fert A, Nguyen Van Dau F, Petroff F, Etienne P, et al. Giant magnetoresistance of $(001) \mathrm{Fe} /(001) \mathrm{Cr}$ magnetic superlattices. Phys Rev Lett. (1988) 61:2472-5. doi: 10.1103/PhysRevLett.61.2472

22. Binasch G, Grünberg P, Saurenbach F, and Zinn $\mathrm{W}$. Enhanced magnetoresistance in layered magnetic structures with antiferromagnetic interlayer exchange. Phys Rev B (1989) 39:4828-30. doi 10.1103/PhysRevB.39.4828

23. Meservey R, and Tedrow PM. Spin-polarized electron tunneling. Phys Rep. (1994) 238:173243. doi: 10.1016/0370-1573(94)90105-8

24. Moodera JS, Kinder LR, Wong TM, and Meservey. R. Large magnetoresistance at room temperature in ferromagnetic thin film tunnel junctions. Phys Rev Lett. (1995) 74:3273-6. doi: 10.1103/PhysRevLett.74.3273

25. Dyakonov MI, and Perel VI. Possibility of orientating electron spins with current. Sov Phys JETP Lett. (1971) 13:467.
26. Kato Y, Myers RC, Gossard AC, and Awschalom DD. Observation of the spin hall effect in semiconductors. Science (2004) 306:1910-3. doi: 10.1126/science. 1105514

27. Wunderlich J, Kaestner B, Sinova J, and Jungwirth T. Experimental observation of the spin-hall effect in a two-dimensional spin-orbit coupled semiconductor system. Phys Rev Lett. (2005) 94:047204. doi: 10.1103/PhysRevLett.94.047204

28. Liu L, Pai, CF, Li Y, Tseng HW, Ralph DC, and Buhrman RA. Spin-torque switching with the giant spin hall effect of tantalum. Science (2012) 336:555-8. doi: 10.1126/science. 1218197

29. Kibble T, and Srivastava A. (eds.). Condensed matter analogues of cosmology, special issue. J Phys Condens Matter (2013) 25:400301. doi: 10.1088/0953-8984/25/40/400301

30. Mott NF. Electrons in transition metals. Adv Phys. (1964) 13:325-422. doi: $10.1080 / 00018736400101041$

Received: 28 November 2013; accepted: 16 December 2013; published online: 27 December 2013.

Citation: Tsymbal EY and Dowben PA (2013) Grand challenges in condensed matter physics: from knowledge to innovation. Front. Physics 1:32. doi: 10.3389/ fphy.2013.00032

This article was submitted to Condensed Matter Physics, a section of the journal Frontiers in Physics. Copyright (c) 2013 Tsymbal and Dowben. This is an open-access article distributed under the terms of the Creative Commons Attribution License (CC BY). The use, distribution or reproduction in other forums is permitted, provided the original author(s) or licensor are credited and that the original publication in this journal is cited, in accordance with accepted academic practice. No use, distribution or reproduction is permitted which does not comply with these terms. 